\title{
AN INVERSE PROBLEMS FOR STURM-LIOUVILLE-TYPE DIFFERENTIAL EQUATION WITH A CONSTANT DELAY
}

\author{
VLADIMIR VLADIČIĆ AND MILENKO PIKULA
}

\begin{abstract}
The topic of this paper is non-self-adjoint second order differential operators with constant delay generated by $-y^{\prime \prime}+q(x) y(x-\tau)$ where potential $q$ is complex-valued function, $q \in L^{2}[0, \pi]$. We establish properties of the spectral characteristics and research the inverse problem of recovering operators from their spectra when $\tau \in\left(\frac{\pi}{2}, \pi\right)$. We prove that the delay and the potential is uniquely determined from two spectrum, firstly when $y(0)=y(\pi)=0$ and secondly when $y(0)=$ $y^{\prime}(\pi)=0$, of those operators. Also we will construct $q$ and $\tau$.
\end{abstract}

\section{INTRODUCTION}

The inverse problems in the spectral theory of operators, especially differential operators, have been studied since the 1930s. This is the first paper in this area [1]. More detailed of this topic can be found in the monograph [5]. A separate chapter of this study deals with the inverse tasks for the boundary problems of the generated equations with a delay. The papers [2], [4], and [6] present the latest results in this field. In this paper we study differential operators of Sturm-Liouville-type generated with second order differential equations with a constant delay. We solve the inverse spectral problem of these operators when $\tau \in\left(\frac{\pi}{2}, \pi\right)$. This means the determining of these operators from their spectra.

The inverse problem of classical Sturm-Liouville operators is fully solved. The solution can be found in [7]. In the problems with constant delay, the methods used for solving of the classical problem (transformation operator method, method of spectral mappings and others) do not give solutions. Because of that, the inverse problem of second order differential operators with constant delay is not solved till today.

2010 Mathematics Subject Classification. 34B24, 34A55.

Key words and phrases. Differential operators with delay,inverse problem, Fourier trigonometric coefficients.

Copyright (c) 2016 by ANUBIH. 
In this paper we study two boundary spectral problems $L_{k}, k=0,1$, generated by

$$
\begin{gathered}
-y^{\prime \prime}(x)+q(x) y(x-\tau)=\lambda y(x), x \in(0, \pi) \\
y(x-\tau)=0, x \in[0, \tau] \\
y^{k}(\pi)=0, k=0,1
\end{gathered}
$$

where $\lambda$ is the spectral parameter, $\tau \in\left(\frac{\pi}{2}, \pi\right)$ is the delay, $q(x)$ is a complexvalued function, such that $q \in L^{2}(0, \pi)$. It is known that the spectrum of $L_{0}, L_{1}$ is countable. We will prove that the delay $\tau$ and the potential $q$ are uniquely determined from the spectrum of $L_{0}$ and $L_{1}$. More precisely, let $\left(\lambda_{n}\right)_{n=1}^{\infty}$ be the eigenvalues of $L_{0}$ and $\left(\mu_{n}\right)_{n=1}^{\infty}$ be the eigenvalues of $L_{1}$. The inverse problem is to determine $q(x)$ and $\tau$ from $\left(\lambda_{n}\right)_{n=1}^{\infty}$ and $\left(\mu_{n}\right)_{n=1}^{\infty}$.

The inverse problem: Given $\left(\lambda_{n}\right)_{n=1}^{\infty}$ and $\left(\mu_{n}\right)_{n=1}^{\infty}$ construct $q$ and $\tau$. In the section spectral properties, we study spectral properties of this problem, and show that it is important to determine the asymptotic behavior of the spectrum. In the section main results, we provide a uniqueness for delay and potential,and also we construct $\tau$ and $q$. We determined the delay and Fourier coefficients of potential from the spectrum of $L_{0}$ and $L_{1}$.

\section{Spectral properties}

Let function $y$ be the solution of the equation (1) under initial condition $y(0)=0$, then function $y$ is unique solution of the integral equation:

$$
y(x)=\frac{1}{z} \int_{0}^{x} q(t) y(t-\tau) \sin z(x-t) d t+C_{1} \sin z x
$$

where $\lambda=z^{2}$.

We will be solving equation (4) along with (2) by steps. For $x \in[0, \tau)$, The solution is:

$$
y(x)=\frac{1}{z} \int_{0}^{x} q(t) y(t-\tau) \sin z(x-t) d t+C_{1} \sin z x=C_{1} \sin z x .
$$

For $x \in[\tau, \pi]$ :

$$
y(x)=\frac{1}{z} \int_{0}^{x} q(t) y(t-\tau) \sin z(x-t) d t+C_{1} \sin z x=\frac{1}{z} \int_{0}^{\tau} q(t) y(t-\tau) \sin z(x-t) d t
$$


$+\frac{1}{z} \int_{\tau}^{x} q(t) y(t-\tau) \sin z(x-t) d t=\frac{C_{1}}{z} \int_{\tau}^{x} q(t) \sin z(t-\tau) \sin z(x-t) d t+C_{1} \sin z x$.

From (5) and (3) we have

$$
F_{0}(z)=\Delta_{0}(\lambda)=y(\pi)=C_{1}\left(\sin z \pi+\frac{1}{z} \int_{\tau}^{\pi} q(t) \sin z(t-\tau) \sin z(\pi-t) d t .\right.
$$

From (5) and $\left(3^{\prime}\right)$ we have

$$
F_{1}(z)=\Delta_{1}(\lambda)=y(\pi)=C_{1}\left(z \cos z \pi+\frac{1}{z} \int_{\tau}^{\pi} q(t) \sin z(t-\tau) \cos z(\pi-t) d t .\right.
$$

Without loss of generality let $C_{1}=1$. The functions $\Delta_{i}(\lambda), i \in 0,1$ is entire in $\lambda$ of order $1 / 2$. It is clear that the set of zeros of function $\Delta_{i}(\lambda)$ is equivalent to the spectrum boundary of spectral problems $L_{i}$, respectively (see[5]). We know that the spectrum of boundary spectral problems $L_{i}$ is countable (see[5]).

Now, if $\left(\lambda_{n}\right)_{n=1}^{\infty}$ is the spectrum of boundary spectral problems $L_{0}$, using (7) by the well-known method (see[2,Ch.1]), we obtain

$$
\lambda_{n}=n^{2}+\frac{\cos \tau n}{\pi} \int_{\tau}^{\pi} q(t) d t+o(1),(n \rightarrow \infty)
$$

The function $\Delta_{0}(\lambda)$, given by (6), is uniquely determined by the spectrum $\left(\lambda_{n}\right)_{n=1}^{\infty}$ of $L_{0}$ with the formulae

$$
\Delta_{0}(\lambda)=\pi \prod_{n=1}^{\infty} \frac{\lambda_{n}-\lambda}{n^{2}}
$$

The function $\Delta_{1}(\lambda)$, given by (7), is uniquely determined by the spectrum $\left(\mu_{n}\right)_{n=1}^{\infty}$ of $L_{1}$ with the formulae

$$
\Delta_{1}(\lambda)=\pi \prod_{n=1}^{\infty} \frac{\mu_{n}-\lambda}{\left(n-\frac{1}{2}\right)^{2}}
$$

(see[6, Lemma 1]).

Because $y(x-\tau)=0, x \in[0, \tau)$ for $L_{i}, i \in 0,1$ without loss of generality we suppose $q(x)=0$ for $x \in[0, \tau)$. 


\section{MAIN RESUlts}

We will first show that the delay is uniquely determined by the spectrum.

Lemma 1. If $\left(\lambda_{n}\right)_{n=1}^{\infty}$ is the spectrum of boundary spectral problems $L$ then the delay $\tau$ is uniquely ordered.

Proof. Since $\tau \in\left(\frac{\pi}{2}, \pi\right)$, there are infinitely many $k \in N$ with property $\operatorname{sink} \tau \neq 0$. From (8) we have

$$
\begin{aligned}
\lim _{k \rightarrow \infty} \frac{\lambda_{k-2}-(k-2)^{2}-\lambda_{k+2}+(k+2)^{2}}{\lambda_{k-1}-(k-1)^{2}-\lambda_{k+1}+(k+1)^{2}} \\
\quad=\lim _{k \rightarrow \infty} \frac{\cos \tau(k-2)-\cos \tau(k+2)}{\cos \tau(k-1)-\cos \tau(k+1)}=\lim _{k \rightarrow \infty} \frac{\sin k \tau \sin 2 \tau}{\sin k \tau \sin \tau}=2 \cos \tau .
\end{aligned}
$$

Finally

$$
\tau=\arccos \left(\frac{1}{2} \lim _{k \rightarrow \infty} \frac{\lambda_{k-2}-(k-2)^{2}-\lambda_{k+2}+(k+2)^{2}}{\lambda_{k-1}-(k-1)^{2}-\lambda_{k+1}+(k+1)^{2}}\right) .
$$

Lemma 2. If $\left(\lambda_{n}\right)_{n=1}^{\infty}$ is spectrum of boundary spectral problems $L_{0}$ then $\int_{\tau}^{\pi} q(t) d t$ is uniquely ordered.

Proof. Because $\tau \in\left(\frac{\pi}{2}, \pi\right)$, there are infinitely many $k \in N$ satisfying $\operatorname{cosk} \tau \neq 0$. From (8) we have

$$
\lim _{k \rightarrow \infty} \frac{\pi\left(\lambda_{k}-k^{2}\right)}{\cos \tau k}=\lim _{k \rightarrow \infty} \frac{\pi\left(k^{2}+\frac{1}{\pi} \cos \tau k \int_{\tau}^{\pi} q(t) d t+o\left(\frac{1}{k^{\frac{1}{2}}}\right)-k^{2}\right)}{\cos \tau k}=\int_{\tau}^{\pi} q(t) d t .
$$

By using $q(x)=0$ for $x \in[0, \tau)$, we obtain $\int_{0}^{\pi} q(t) d t=\int_{\tau}^{\pi} q(t) d t$. We have proved that the first Fourier coefficient of the potential $q$ on the $[0, \pi]$ is uniquely determined from the spectrum of $L_{0}$.

Now we will prove that the other Fourier coefficients of the potential $q$ are also uniquely determined. We introduce the notation $a_{n}=\int_{0}^{\pi} q(t) \cos 2 n t d t$ and $b_{n}=\int_{0}^{\pi} q(t) \sin 2 n t d t$. By using the above described observations, we finally come to our main result.

Theorem 1. If $\left(\lambda_{n}\right)_{n=1}^{\infty}$ is the spectrum of boundary spectral problems, $L_{0}$ and $\left(\mu_{n}\right)_{n=1}^{\infty}$ is the spectrum of boundary spectral problems $L_{1}$, then Fourier coefficients $a_{n}$ and $b_{n}$, of the potential $q$, are uniquely determined for every $n \in N$. 
Proof. From (6) and (7)

$$
\begin{gathered}
F_{0}(z)=\sin z \pi+\frac{1}{z} \int_{\tau}^{\pi} q(t) \sin z(t-\tau) \sin z(\pi-t) d t \\
F_{1}(z)=z \cos z \pi+\frac{1}{z} \int_{\tau}^{\pi} q(t) \sin z(t-\tau) \cos z(\pi-t) d t
\end{gathered}
$$

We transform product of trigonometric functions to sum and we have

$$
\begin{gathered}
F_{0}(z)=\sin z \pi+\frac{\cos z(\pi-\tau)}{2 z} \int_{\tau}^{\pi} q(t) d t+ \\
\frac{\cos z(\pi+\tau)}{2 z} \int_{\tau}^{\pi} q(t) \cos 2 z t d t+\frac{\sin z(\pi+\tau)}{2 z} \int_{\tau}^{\pi} q(t) \sin 2 z t d t \\
F_{1}(z)=z \cos z \pi+\frac{\sin z(\pi-\tau)}{2 z} \int_{\tau}^{\pi} q(t) d t+ \\
\frac{\cos z(\pi+\tau)}{2 z} \int_{\tau}^{\pi} q(t) \sin 2 z t d t-\frac{\sin z(\pi+\tau)}{2 z} \int_{\tau}^{\pi} q(t) \cos 2 z t d t
\end{gathered}
$$

Now we put $z=n, n \in N$ and using $a_{n}=\int_{0}^{\pi} q(t) \cos 2 n t d t$ and $b_{n}=$ $\int_{0}^{\pi} q(t) \sin 2 n t d t$ we have

$$
\begin{gathered}
F_{0}(n)-\frac{\cos n(\pi-\tau)}{2 n} \int_{\tau}^{\pi} q(t) d t=\frac{\cos n(\pi+\tau)}{2 n} a_{n}+\frac{\sin n(\pi+\tau)}{2 n} b_{n} \\
F_{1}(n)-n(-1)^{n}-\frac{\operatorname{sinn}(\pi-\tau)}{2 n} \int_{\tau}^{\pi} q(t) d t=\frac{\cos n(\pi+\tau)}{2 n} b_{n}-\frac{\operatorname{sinn}(\pi+\tau)}{2 n} a_{n}
\end{gathered}
$$

Finally,

$$
\begin{aligned}
& a_{n}=2 n \operatorname{cosn}(\pi+\tau)\left(F_{0}(n)-\frac{\cos n(\pi-\tau)}{2 n} \int_{\tau}^{\pi} q(t) d t\right)- \\
& \operatorname{sinn}(\pi+\tau)\left(F_{1}(n)-n(-1)^{n}-\frac{\operatorname{sinn}(\pi-\tau)}{2 n} \int_{\tau}^{\pi} q(t) d t\right. \\
& b_{n}=2 n\left(\operatorname{sinn}(\pi+\tau)\left(F_{0}(n)-\frac{\operatorname{cosn}(\pi-\tau)}{2 n} \int_{\tau}^{\pi} q(t) d t\right)+\right.
\end{aligned}
$$




$$
\left.\cos n(\pi+\tau)\left(F_{1}(n)-n(-1)^{n}-\frac{\operatorname{sinn}(\pi-\tau)}{2 n} \int_{\tau}^{\pi} q(t) d t\right)\right)
$$

Since $\tau, \int_{\tau}^{\pi} q(t) d t$ determinate from spectrum (Lemma1 and Lemma2) and functions $F_{0}, F_{1}$ also determinate from spectrum we completed the proofing of the theorem.

With Theorem 1 we have proved the uniqueness of potential, since $q \in$ $L^{2}[0, \pi]$, also we construct potential $q$ with

$$
q(x)=\sum_{n=-\infty}^{n=+\infty} c_{n} e^{2 i n x}
$$

where $c_{n}=\frac{1}{\pi} a_{n}-\frac{i}{\pi} b_{n}$

\section{REFERENCES}

[1] Ambarzumjan V., Uber eine Frage der Eigenwerttheorie, Zeitshr.fr Physik, -Bd.53., -S. , 690-695 (1929)

[2] Bayramov A., Ozturk Uslub S., Kizilbudak Caliskan S., Computation of eigenvalues and eigenfunction of a discontinuous boundary value problem with retarded argument, Appl. Math. Comput. 191 , 592-600(2007)

[3] Borg S., Eine Umkehrung der Sturm-Liouvillschen Eigenwertaufgabe, Acta Math. Bd.78.No.1. -S , 1-96 (1946)

[4] Chuan-Fu Yang, Trace and inverse problem of a discontinuous Sturm-Liouville operator with retarded argument, J. Math. Anal. Appl. 395,30-41(2012)

[5] Freiling G. and Yurko V., Inverse Sturm-Liouville problems and their applications Nova Science Publishers, Inc.Huntigton, NewYork, (2008)

[6] Freiling G. and Yurko V, Inverse problems for Sturm-Liouville diferential operators with a constant delay, Appl. Math. Lett. 25, No. 11,1999-2004(2012)

[7] Levitan B.M. and Sargsjan I.S., Sturm-Liouville and Dirac Operators, Nauka, Moscow,1988; English transl.:Kluwer Academic Publishers, Dordrecht, (1991)

(Received: July 23, 2015)

(Revised: October 8, 2015)
Vladimir Vladičić and Milenko Pikula

University East Sarajevo

Department of Mathematics, Informatics and Physics

Alekse Šantića 1, East Sarajevo

Bosnia and Herzegovina

vladimir.vladicic@ffuis.edu.ba

mpikula@paleol.net 